\title{
Occlusion de la veine rétinienne centrale secondaire à un traumatisme oculaire chez un patient jeune : Rapport de cas
}

\author{
Ailia Ahmed, Do \\ Vision nationale
}

\author{
Justin Simbulan, Do \\ Le Collège d'optométrie de \\ l'Université de Houston
}

Bhagya Segu, DO, MPH, FAAO

Centre médical pour vétérans Michael E. DeBakey

Angelina Tran, DO, FAAO Centre médical pour vétérans Michael E. DeBakey

\begin{abstract}
Résumé
Les occlusions de la veine rétinienne centrale (OVRC) d'étiologie traumatique ou chez les patients jeunes sont rares. Cet article décrit le cas d'un homme asiatique de 33 ans qui s'est présenté avec une plainte principale de vision périphérique floue et de photophobie dans l'œil gauche, une semaine après avoir subi un traumatisme oculaire contondant. Il a reçu un diagnostic d'OVRC traumatique fondé sur ses antécédents et les résultats de l'examen.
\end{abstract}

MOTS CLÉS :

OVRC, traumatisme contondant

\section{INTRODUCTION}

Après la rétinopathie diabétique, l'occlusion de la veine rétinienne est la deuxième cause la plus courante de troubles vasculaires rétiniens. Les occlusions des branches veineuses rétiniennes sont plus fréquentes, avec une prévalence dans la population américaine de 0,5 à 1,2\%, par rapport aux OVRC, qui se produisent avec une prévalence de 0,1 à $0,5 \%$. Les OVRC se présentent généralement comme une perte de vision soudaine, unilatérale et indolore chez les personnes âgées de 50 ans ou plus souffrant de vasculopathies. L'OVRC est rare chez les personnes de 40 ans ou moins, et pour ces personnes, des étiologies telles que l'utilisation de contraceptifs oraux, une collagénose avec manifestations vasculaires, le syndrome d'immunodéficience acquise (SIDA), la déficience en protéine S/protéine $\mathrm{C}$, la déficience en antithrombine III, la déficience en facteur XII, le syndrome des anticorps antiphospholipides ou la résistance à la protéine $\mathrm{C}$ activée doivent être prises en compte ${ }^{2}$. Dans de rares cas, une OVRC peut être une manifestation clinique de traumatisme oculaire.

\section{RAPPORT DE CAS}

\section{Historique}

Un homme asiatique de 33 ans s'est présenté en se plaignant d'une vision périphérique floue et d'une photophobie dans l'œil gauche depuis une semaine. Il a signalé un traumatisme récent survenu deux semaines auparavant; il a reçu un coup à l'œil gauche pendant la pratique de Jiu-Jitsus. Le flou visuel périphérique et la photophobie se sont produits une semaine après la blessure. Les antécédents oculaires du patient ont révélé un astigmatisme myopique combiné à des antécédents de kératomileusis in situ au laser (LASIK). Les antécédents médicaux du patient n'avaient rien de particulier et il ne prenait pas de médicaments. Il n'avait aucune allergie connue.

\section{Examen clinique}

La meilleure acuité visuelle corrigée (MAVC) du patient était de 20/20 OD, OS. Ses pupilles étaient égales en taille, rondes et réactives à la lumière, sans déficit pupillaire afférent (DPA). Les mouvements extraoculaires n'étaient pas restreints et en confrontation, le champ visuel était entier OU. L'examen à la lampe à fente du segment antérieur n'a rien révélé de marquant, à l'exception de cellules traces OS. On n'a relevé aucune ecchymose, aucun œè̀me ou aucune anomalie des annexes oculaires OU. Sa pression intraoculaire (PIO) mesurée au tonomètre de Goldmann était de $10 \mathrm{mmHg}$ OD et $11 \mathrm{mmHg}$ OS. Lors de l'examen du fond d'œil dilaté (EFD), l'œil droit n'a rien révélé de mar- 
quant et l'œil gauche présentait un œdème papillaire avec hémorragie péripapillaire, hémorragie parafovéale diffuse en forme de flammèche dans la macula et hémorragie en forme de flammèche et microanévrisme dans les quatre quadrants périphériques de la rétine (figures 1 et 2). Il s'agissait d'une présentation atypique de l'OVRC, car l'hémorragie dans ce cas était concentrée autour du disque, avec une hémorragie moins grave loin du pôle postérieur. Cela peut être dû à une pression importante sur le nerf optique lui-même en raison du traumatisme, par opposition à l'étiologie vasculopathique d'autres OVRC. La tomographie par cohérence optique (TCO) de la macula a démontré une macula plate et intacte sans liquide sous-rétinien ou intrarétinien OU (figure 3). La TCO de la couche de fibres nerveuses rétiniennes se situait dans les limites normales OD et était élevée à 360 degrés OS, ce qui a confirmé l'œè̀me péripapillaire (figure 4). La tension artérielle du patient au bureau était de $123 / 73 \mathrm{mmHg}$.

Figures 1 et 2 : Pôles postérieurs droit et gauche avec hémorragies importantes de l'œil gauche
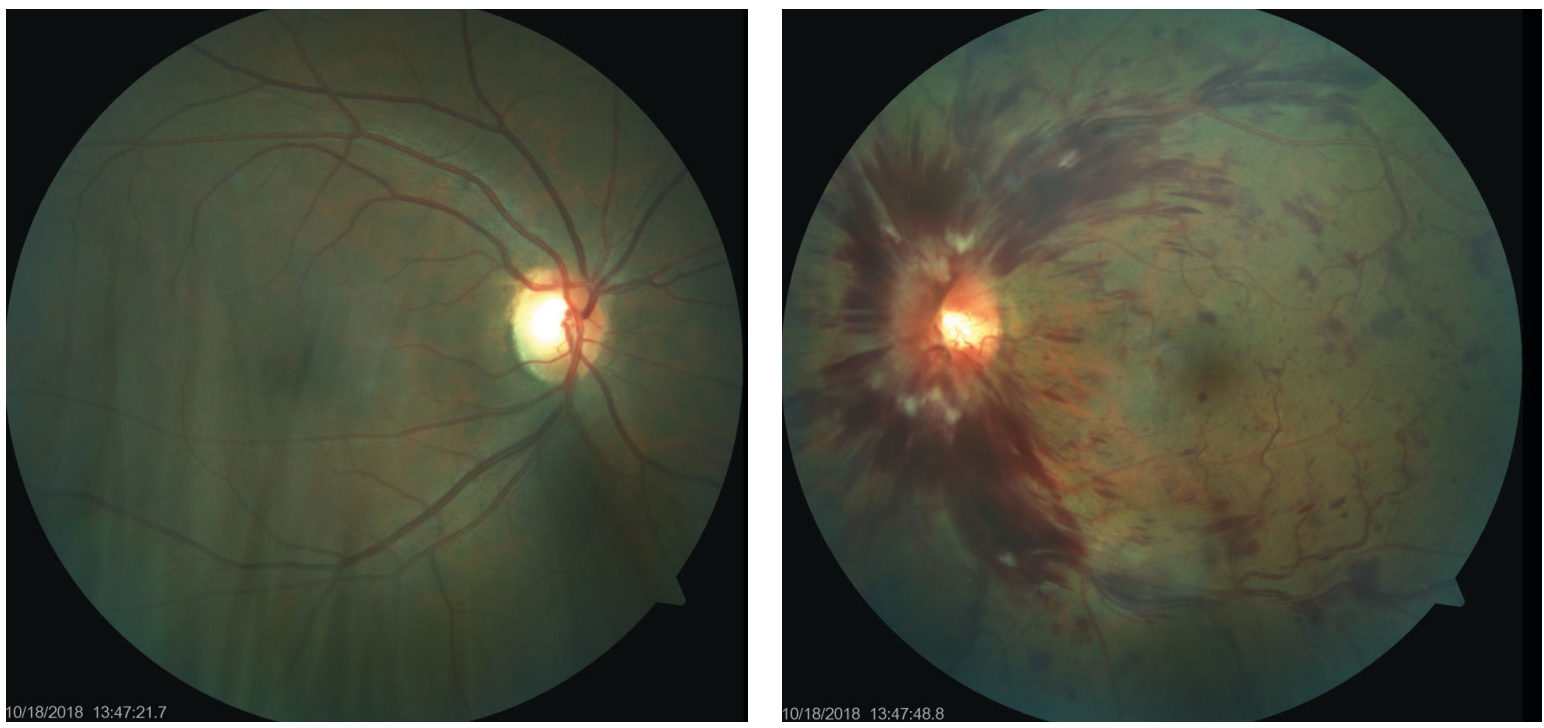

Figure 3 : TCO maculaires avec légère augmentation de l'épaississement maculaire de l'œil gauche
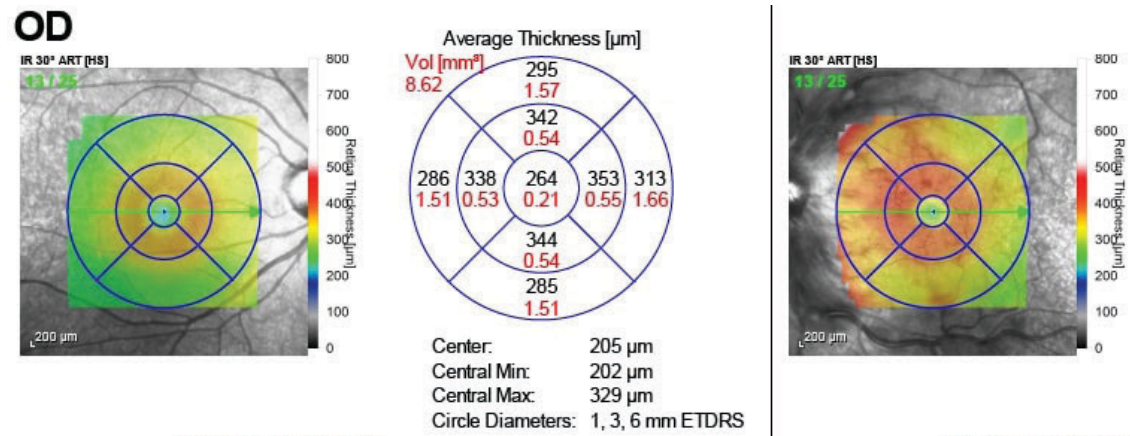

Average Thickness $[\mu \mathrm{m}]$

OS
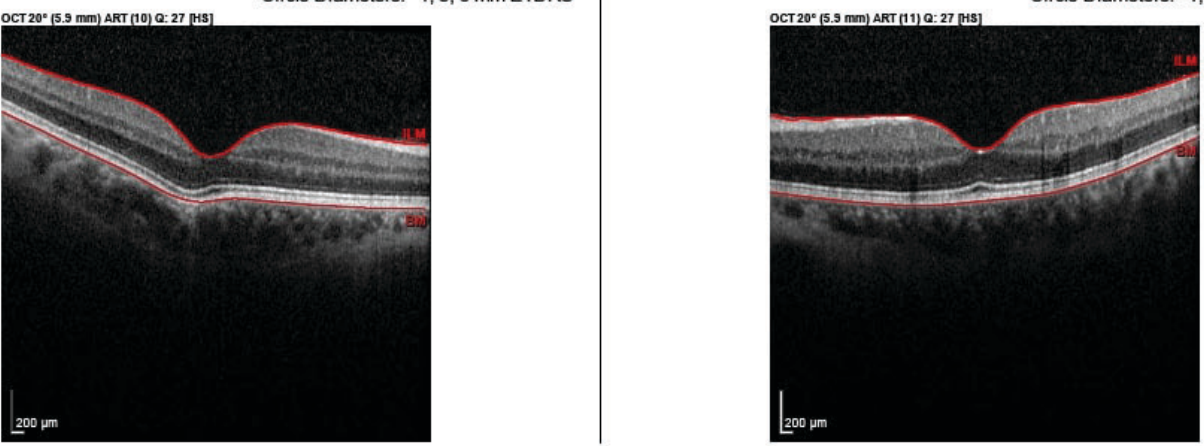
Figure 4 : TCO des disques du nerf optique avec œdème papillaire de l'œil gauche

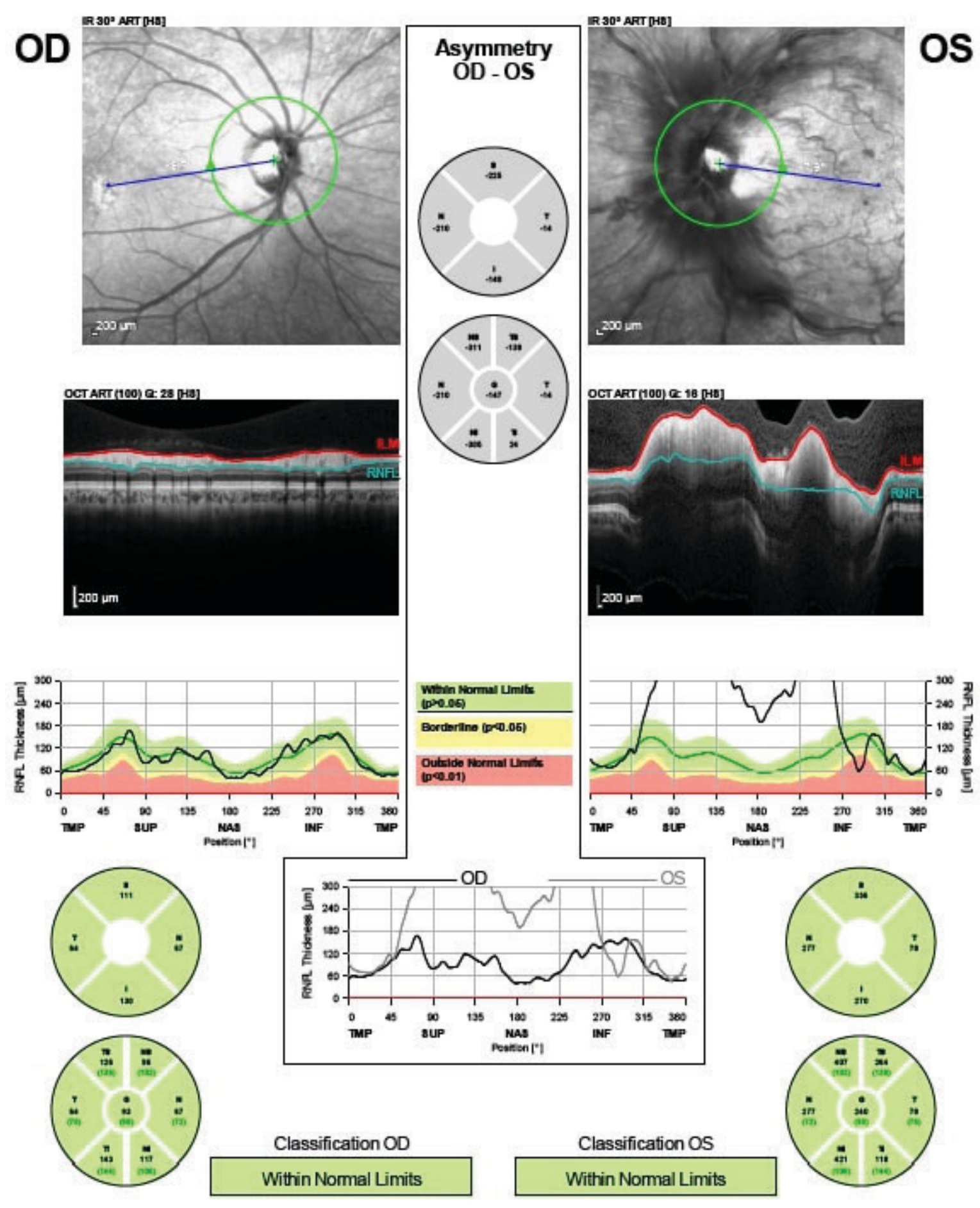




\section{Diagnostic et traitement}

Compte tenu des récents antécédents de traumatisme et des résultats de l'examen, le patient a reçu un diagnostic d'OVRC traumatique avec une iritis traumatique légère OS. Le cas a été examiné par un spécialiste de la rétine qui a accepté le diagnostic et l'étiologie, en attendant les résultats de laboratoire pour exclure la dyscrasie sanguine systémique. Le patient a été informé que le problème se résoudrait de lui-même et on lui a conseillé d'éviter les activités vigoureuses. On lui a prescrit de l'acétate de prednisolone trois fois par jour per os pour l'iritis et il devait revenir deux semaines plus tard pour une vérification de la PIO, une gonioscopie, un EFD, une TCO de la macula et une TCO de la CFNR.

\section{Suivi et analyses supplémentaires}

Le patient a fait l'objet d'un suivi deux semaines après son premier examen, au cours duquel on a observé la résolution de la réaction de la chambre antérieure. La dose de stéroïdes topiques a donc été réduite graduellement et le patient a été vu à intervalles mensuels afin de détecter l'apparition d'une néovascularisation ou l'aggravation des signes ou symptômes cliniques. Au cours de ses visites de suivi mensuelles, la MAVC du patient est demeurée de 20/20 OD, OS et il a signalé subjectivement une amélioration et une résolution des symptômes de flou périphérique OS. Une gonioscopie a été effectuée à toutes les visites de suivi et aucune récession d'angle ou néovascularisation n'a été notée. Le patient a démontré une résolution graduelle des observations du fond de l'œil et un gonflement de la papille à chaque visite de suivi, avec une amélioration importante cinq mois après la présentation initiale (figures 5 et 6 ). Des analyses de laboratoire supplémentaires ont écarté d'autres étiologies qui pourraient expliquer la présence d'une OVRC chez un patient jeune (tableau 1).

Figure 5 : Pôle postérieur gauche avec résolution des hémorragies rétiniennes

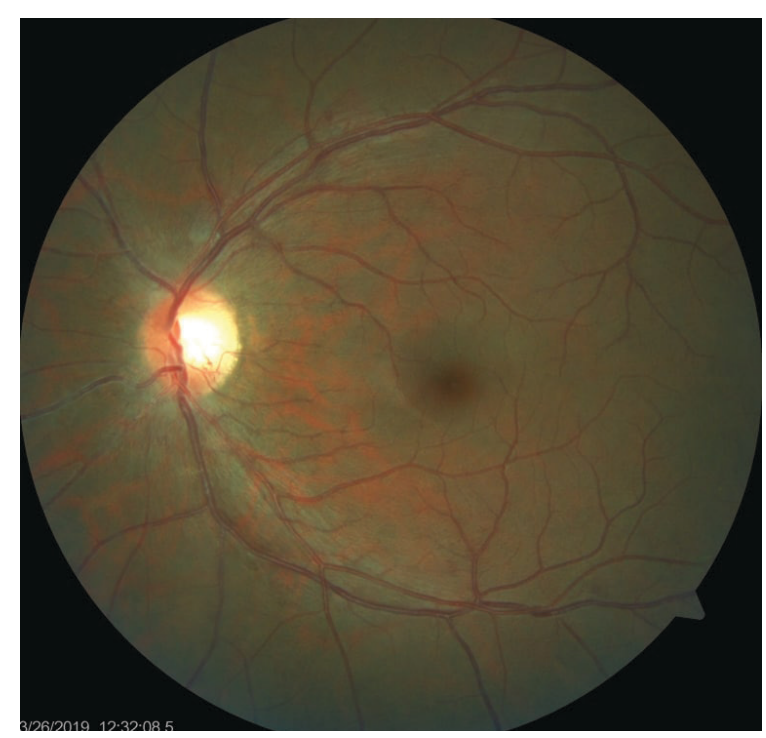

\section{DISCUSSION}

Aux États-Unis, les traumatismes oculaires sont responsables d'environ 30000 visites au service des urgences chaque année $^{3}$. Les constatations oculaires d'étiologie traumatique courantes comprennent la contusion, l'ecchymose, l'hyphème, la cataracte, le glaucome, l'hémorragie vitréenne, l'hémorragie rétinienne et l'œedème rétinien de Berlin. L'OVRC traumatique dont il est question dans ce rapport de cas est une manifestation très rare du traumatisme oculaire et très peu d'exemples ont été décrits dans la littérature.

Les signes cliniques d'OVRC comprennent des hémorragies rétiniennes superficielles et profondes dans les quatre quadrants de la rétine, un engorgement et une tortuosité des vaisseaux, un gonflement papillaire, des nodules cotonneux et un œdème maculaire cystoïde ${ }^{1}$. Les OVRC surviennent généralement chez des personnes de plus de 50 ans présentant des facteurs de risque tels que l'hypertension, les maladies vasculaires et l'ascendance afro-américaine. En outre, les patients souffrant de lésions aux organes cibles dues au diabète sucré courent un risque accru d'OVRC par rapport aux patients atteints de diabète sucré sans complication ${ }^{4}$. Parmi les autres causes moins courantes, on peut citer l'utilisation de contraceptifs oraux, la collagénose avec manifestations vasculaires, le SIDA, la déficience en protéine $\mathrm{S}$, la déficience en protéine $\mathrm{C}$, la déficience en antithrombine III, la déficience en facteur XII, le syndrome des anticorps antiphospholipides ou la résistance à la protéine $\mathrm{C}$ activée. 
Figure 6 : TCO du disque du nerfoptique gauche avec résolution de l'oedème papillaire
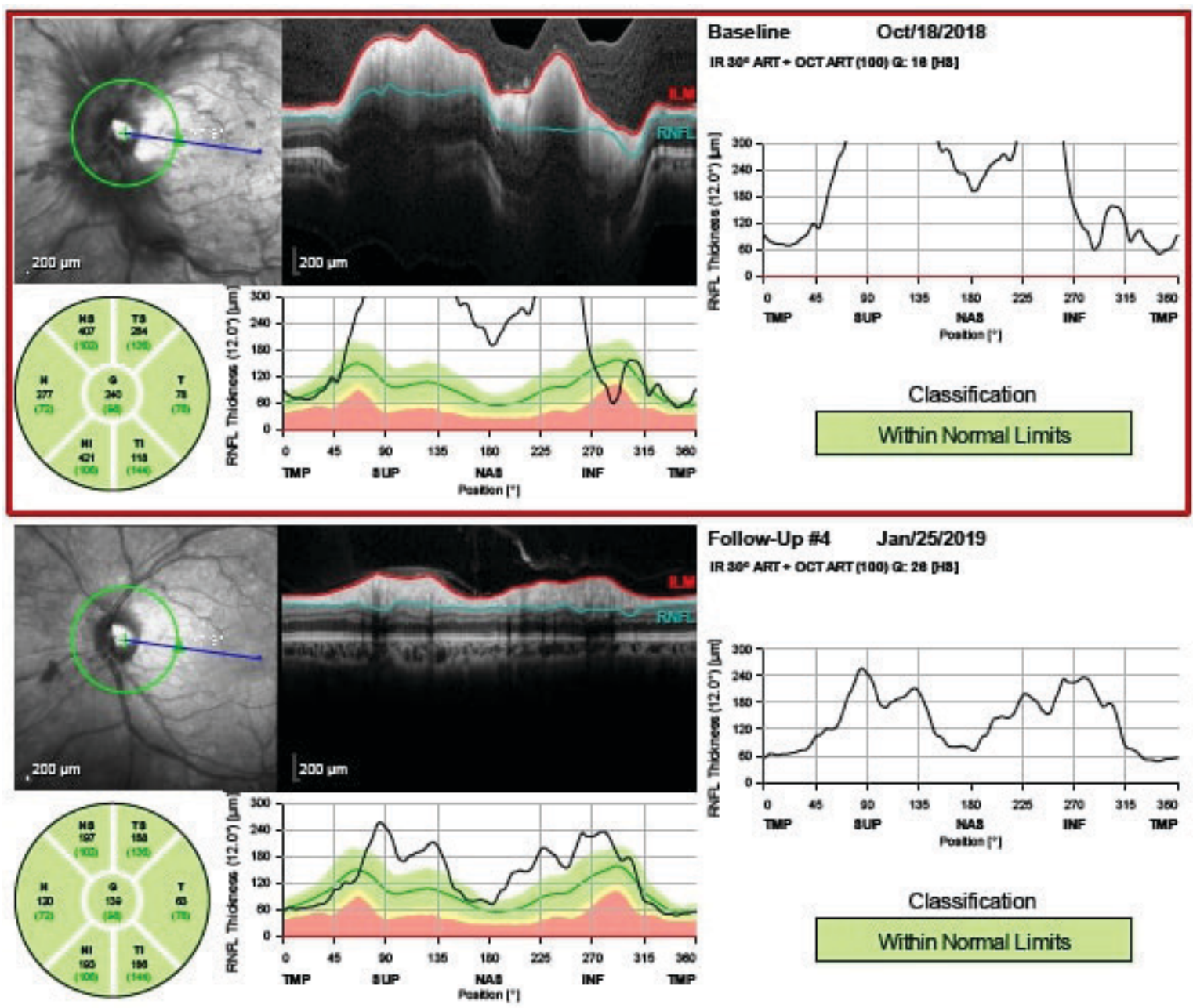

Follow-Up \#4 Jan/25/2019

IR 300 AFT + OCT ART [100) \&: 28 0H:]
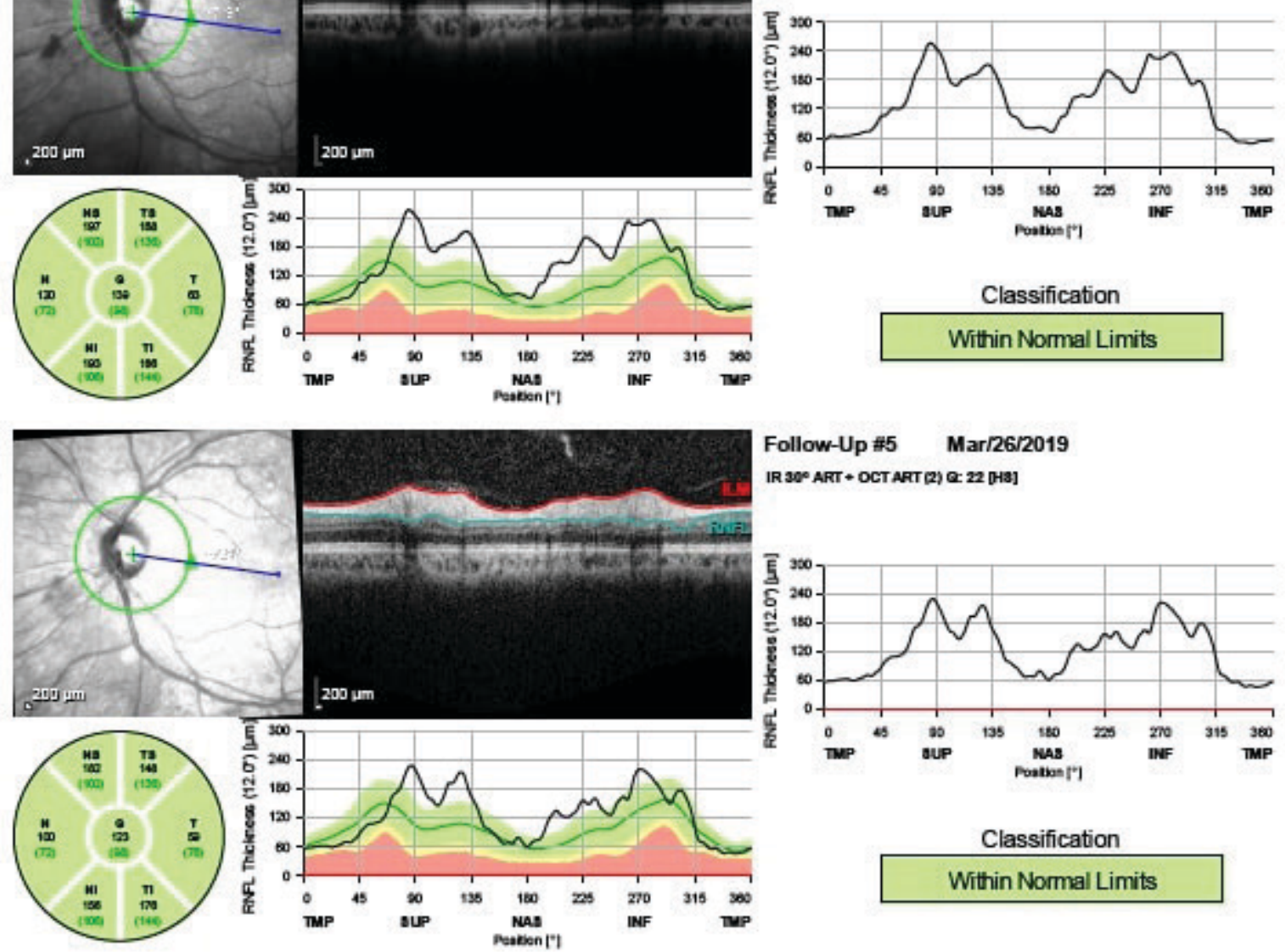

Follow-Up \#5 Mar/26/2019

IR 300 ART + OCT ART [2] \&: 22 [H3]

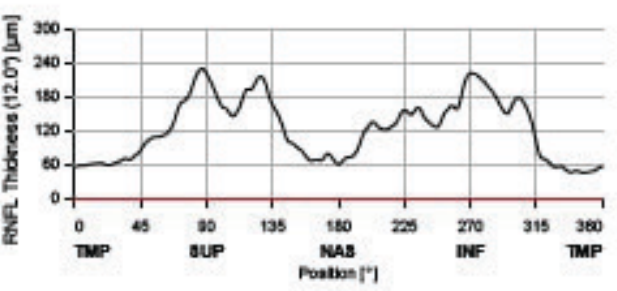

Classification

Within Normal Limits 
Tableau 1: Analyses de laboratoire indiquant qu'il n'y a pas d'étiologies sous-jacentes à l'OVRC chez un jeune patient

\begin{tabular}{|c|c|c|}
\hline Test & Résultat & Plage de référence \\
\hline VS & $4 \mathrm{~mm} / \mathrm{h}$ & $0-22 \mathrm{~mm} / \mathrm{h}$ \\
\hline Homocystéine & $9,8 \mu \mathrm{mol} / \mathrm{L}$ & $0,0-15,0 \mu \mathrm{mol} / \mathrm{L}$ \\
\hline AAN & Positif* & $<1: 40$ \\
\hline RPR & Non réactif & Non réactif \\
\hline MHA-TP & Non réactif & Non réactif \\
\hline Cholestérol LDL & $145,2 \mathrm{mg} / \mathrm{dL}$ & $0-130 \mathrm{mg} / \mathrm{dL}$ \\
\hline Cholestérol total & $225 \mathrm{mg} / \mathrm{dL}$ & $0-200 \mathrm{mg} / \mathrm{dL}$ \\
\hline HDL & $59 \mathrm{mg} / \mathrm{dL}$ & $29-72 \mathrm{mg} / \mathrm{dL}$ \\
\hline Triglycérides & $102 \mathrm{mg} / \mathrm{dL}$ & $10-170 \mathrm{mg} / \mathrm{dL}$ \\
\hline Numération leucocytaire & $5,210^{3} / \mu \mathrm{L}$ & $3,5-10,010^{3} / \mu \mathrm{L}$ \\
\hline Érythrocytes & $5,410^{6} / \mu \mathrm{L}$ & $4,0-5,910^{6} / \mu \mathrm{L}$ \\
\hline Hémoglobine & $15,2 \mathrm{~g} / \mathrm{dL}$ & $12,0-18,0 \mathrm{~g} / \mathrm{dL}$ \\
\hline $\mathrm{HCT}$ & $46,0 \%$ & $36,0-52 \%$ \\
\hline $\mathrm{MCV}$ & $85,9 \mathrm{fL}$ & 81-100 fL \\
\hline $\mathrm{MCH}$ & $28,3 \mathrm{pg}$ & $27-35 \mathrm{pg}$ \\
\hline MCHC & $33,0 \mathrm{~g} / \mathrm{dL}$ & $33,0-36,0 \mathrm{~g} / \mathrm{dL}$ \\
\hline RDW & $13,0 \%$ & $12,3-16,4 \%$ \\
\hline Numération plaquettaire & $22210^{3} / \mu \mathrm{L}$ & $150-45010^{3} / \mu \mathrm{L}$ \\
\hline MPV & $9,1 \mathrm{fL}$ & 7,4-10,4 fL \\
\hline \% Neutrophiles & $61,5 \%$ & $45,0-75,0 \%$ \\
\hline \% Lymphocytes & $28,0 \%$ & $18,0-43,0 \%$ \\
\hline$\%$ Monocytes & $7,3 \%$ & $2,0-14,0 \%$ \\
\hline \% Éosinophiles & $2,6 \%$ & $0,0-8,0 \%$ \\
\hline \% Basophiles & $0,6 \%$ & $0,0-2,0 \%$ \\
\hline Neutrophiles (\#) & $3,2010^{3} / \mu \mathrm{L}$ & $1,6-7,510^{3} / \mu \mathrm{L}$ \\
\hline Lymph (\#) & $1,5010^{3} / \mu \mathrm{L}$ & $0,6-4,310^{3} / \mu \mathrm{L}$ \\
\hline Mono (\#) & $0,4010^{3} / \mu \mathrm{L}$ & $0,1-1,410^{3} / \mu \mathrm{L}$ \\
\hline Éosi (\#) & $0,1010^{3} / \mu \mathrm{L}$ & $0,0-0,810^{3} / \mu \mathrm{L}$ \\
\hline Baso (\#) & $0,0010^{3} / \mu \mathrm{L}$ & $0,0-0,210^{3} / \mu \mathrm{L}$ \\
\hline PT & 13,1 secondes & $12,0-14,7$ secondes \\
\hline PTT & 33,4 secondes & 23,6-36,6 secondes \\
\hline Anticardiolipine AB, IgG & $<9 \mathrm{GPL} \mathrm{U} / \mathrm{mL}$ & 0-14 GPL U/mL \\
\hline Anticardiolipine AB, IgM & < 9 MPL U/mL & 0-12 MPL U/mL \\
\hline
\end{tabular}

* AAN positif à une concentration de 1:40, à laquelle certains sujets de la population normale sont testés positifs sans problèmes systémiques. L’AAN n’a pas donné de résultats positifs à des concentrations plus diluées. 
Il y a deux catégories d'OVRC : non-ischémique et ischémique. Une OVRC ischémique est définie comme l'observation d'une non-perfusion capillaire de plus de 10 diamètres de disque sur un angiogramme à la fluorescéine $(\mathrm{AF})^{2}$. Chez les patients souffrant d'une OVRC ischémique, des niveaux élevés de protéines liés à l'hypoxie, l'angiogenèse et l'inflammation augmentent la probabilité d'une néovascularisation'. Le pronostic visuel de l'OVRC peut varier de 20/20 à la détection des mouvements de la main. Le trouble de la vision due à l'hémorragie importante observée au moment de la visite initiale peut s'améliorer à mesure que l'hémorragie se résorbe, ou, à l'inverse, la perte de vision peut s'aggraver en raison d'une non-perfusion rétinienne prolongée. La non-perfusion peut par la suite entraîner une production accrue du facteur de croissance endothélial vasculaire (VEGF), ce qui peut entraîner un œdème maculaire et une néovascularisation ${ }^{5}$. L'OVRC de notre patient a été diagnostiquée comme non ischémique en raison de l'absence d'une perte de vision grave et de l'absence d'un déficit pupillaire afférent (DPA). Une AF n’a pas été demandée en raison du faible degré de suspicion d'étiologie ischémique dans cette affaire.

Les OVRC traumatiques sont rares dans la littérature. Selon Hayreh et coll., 10 personnes sur 680 ont une OVRC après un traumatisme contondant ${ }^{6}$. El Asri et coll. ont rapporté le cas d'un homme de 35 ans qui a subi un traumatisme oculaire dû à une blessure par explosion dans une zone de conflit et qui s'est présenté avec une baisse de vision soudaine attribuable à une $\mathrm{OVRC}^{7}$. Ce cas diffère de celui de notre patient, car l'homme a constaté une baisse de sa vision et une analyse plus poussée a montré une mutation G20210A du gène du facteur II. Notre patient n'a pas encore subi de tests génétiques, et il est possible qu'il ait une mutation génétique qui le prédispose à l'apparition d'une OVRC. Alvarez et Noble ont décrit un cas d'OVRC associée à une occlusion de l'artère rétinienne centrale (OARC) chez un patient de 28 ans qui a reçu un coup dans l'œil plutôt qu'une OVRC sans occlusion artérielle comme dans le cas de notre patient ${ }^{8}$.

Dans un cas typique d'OVRC, on croit que la pathogenèse implique une obstruction de l'écoulement veineux de la rétine centrale. Différents facteurs tels que les changements vasculaires, les tendances hématologiques et thrombotiques, et la susceptibilité anatomique locale peuvent également contribuer au développement d'une OVRC1. Notre patient a subi un traumatisme contondant à l'œil en pratiquant le Jiu-Jitsu, ce qui a entraîné une déformation mécanique aiguë de l'œil et l'effondrement subséquent de la veine rétinienne centrale contre la lamina cribrosa. Par ailleurs, une augmentation de la PIO peut créer une plus grande différence de pression à l'endroit où la veine sort de l'œil. Toutefois, cette étiologie a été écartée parce que notre patient avait des PIO normales et symétriques, sans hyphène et sans récession d'angle par gonioscopie. Enfin, les anomalies congénitales, comme l'augmentation de la tortuosité vasculaire ou une configuration anatomique anormale, peuvent avoir une incidence sur l'hémodynamique de l'écoulement veineux et prédisposer la veine rétinienne centrale à la thrombose ${ }^{9}$. Notre patient avait une OVRC avec développement progressif probable d'un œedème papillaire. C'est peut-être pourquoi ses symptômes de flou visuel périphérique n'étaient pas aigus et ne se sont pas manifestés qu’après une semaine.

Chez les jeunes adultes, le cours naturel d'une OVCR traumatique est la résolution spontanée sur une période de trois à six mois, avec une diminution minimale de la meilleure acuité visuelle corrigée. Fong et Schatz ont passé en revue 488 cas et ont signalé que ces patients se rétablissent généralement en 12,2 semaines en moyenne $\mathrm{e}^{10}$. En ce qui concerne le traitement, l'étude SCORE2 (Study of Comparative Treatments for Retinal Vein Occlusion 2) a établi que les trois agents anti-VEGF (aflibercept, bévacizumab et ranibizumab) sont efficaces dans le traitement de l'œdème maculaire ${ }^{11}$. Le pronostic de l'OVRC dépend généralement de son caractère non ischémique ou ischémique. Les OVRC non ischémiques ont généralement un meilleur résultat visuel final.

\section{CONCLUSION}

Bien que les OVRC se produisent habituellement dans la population vasculopathique âgée de 50 ans et plus, ce rapport de cas décrit les séquelles inhabituelles d'une OVRC chez un patient jeune à la suite d'un traumatisme contondant. En tant que fournisseurs de soins oculovisuels, nous devons être conscients des complications courantes et des manifestations plus rares des traumatismes oculaires. Des antécédents de cas complets et une prise en charge rapide et appropriée devraient aider à prévenir d'autres complications oculaires.

AUTEUR-RESSOURCE

Ailia Ahmed, DO, Email: ailia72@gmail.com 


\section{RÉFÉRENCES}

1. McAllister IL. Central retinal vein occlusion: a review. Clin Exp Ophthalmol 2012;40:48-58.

2. Friedman NJ, Kaiser PK, Pineda R. The Massachusetts Eye and Ear Infirmary Illustrated Manual of Ophthalmology. China: Elsevier, 2014.

3. Canner JK, Haring RS, Schneider EB, et al. Epidemiology of sports-related eye injuries in the United States. JAMA Ophthalmol 2016;134(12):1382-90.

4. Comer GM, Stein JD, Stem MS, et al. A longitudinal analysis of risk factors associated with central retinal vein occlusion. Ophthalmology 2013;120:362-70.

5. Agostini HT, Ehlken C, Feltgen N, et al. Increased expression of angiogenic and inflammatory proteins in the vitreous of patients with ischemic central retinal vein occlusion. PLoS ONE 2015;10(5):1-15.

6. Hayreh SS, Podhajsky PA, Zimmerman MB. Natural history of visual outcome in central retinal vein occlusion. Ophthalmology 2011;118:119-33.
7. El Asri F, Fenolland JR, Giraud JM, et al. [A rare trauma-associated cause of central retinal vein occlusion in a young subject.] J Fr Ophtalmol 2012;35(6):426-31. (in French)

8. Alvarez EV, Noble MJ. Combined occlusion of the central retinal artery and central retinal vein following blunt ocular trauma: a case report. Br J Opthalmol 1987;71:834-6.

9. Cankaya C, Cumurcu T, Demirel S, et al. Traumatic optic neuropathy and central retinal artery occlusion following blunt ocular trauma. J Clin Med Res 2011;3(1):55-7.

10. Fong ACO, Schatz $\mathrm{H}$. Central retinal vein occlusion in young adults. Surv Ophthalmol 1993;37:393-417.

11. Bressler NM. Treatment of macular edema due to central retinal vein occlusion: another score for repackaged bevacizimab. JAMA 2017 May 23;317(20):2067-9. 